\title{
Green tea consumption and the management of adrenal stress hormones in adolescents who stutter
}

\author{
ABDULAZIZ ALMUDHI ${ }^{1,2}$ and SAMI A. GABR ${ }^{3}$ \\ ${ }^{1}$ Department of Medical Rehabilitation Sciences, College of Applied Medical Sciences; \\ ${ }^{2}$ Speech Language Pathology Unit, King Khalid University, Abha 61481, Saudi Arabia; \\ ${ }^{3}$ Department of Anatomy and Embryology, Faculty of Medicine, Mansoura University, Mansoura 35516, Egypt
}

Received December 12, 2021; Accepted February 14, 2022

DOI: $10.3892 /$ br.2022.1515

\begin{abstract}
Green tea and its polyphenolic compounds have been shown to exert positive effects in individuals with psychological disorders. The protective role of green tea against stuttering or its related consequences, depression, anxiety and stress, were evaluated in adolescents with moderate stuttering (MS). A total of 60 adolescents aged (12-18) years old were enrolled in this study. Patients were classified according to standardized test material Stuttering Severity Instrument, 4th Edition was used to estimate the severity of stuttering; participants were classified into two groups: a normal healthy group ( $\mathrm{n}=30)$ and a MS group $(\mathrm{n}=30)$. The Depression Anxiety Stress Scale and General Health Questionnaire were used to estimate the degree of depression, anxiety and stress as well as general mental health. The physiological profile of stress hormones, as a measure of the response to green tea response, was also measured amongst participants. Adrenal stress hormones cortisol, dehydroepiandrosterone (DHEA), acetylcholine (ACTH), corticosterone and the cortisol:DHEA ratio were assayed. In addition, the constituent green tea polyphenols and their quantities were determined using liquid chromatography analysis. Decaffeinated green tea was administered six cups/day for 6 weeks, and this significantly improved the depression, anxiety, stress and mental health consequences associated with stuttering in adolescents. In addition, increased consumption of green tea significantly reduced elevated levels of adrenal stress hormones; cortisol, DHEA, ACTH and corticosterone, and increased the cortisol:DHEA ratio in the control and adolescents who stuttered. The data showed that drinking six cups of decaffeinated green tea, which is enriched in catechins $(1,580 \mathrm{mg})$ and other related polyphenols, was
\end{abstract}

Correspondence to: Dr Sami A. Gabr, Department of Anatomy and Embryology, Faculty of Medicine, Mansoura University, 60 Elgomhouria Street, Mansoura 35516, Egypt

E-mail: dr.samigabr@gmail.com

Key words: decaffeinated green tea, green tea polyphenols, catechins, stuttering, adolescents, liquid chromatography, adrenal stress hormones sufficient to improve the consequences of mental health associated with stuttering in younger aged individuals.

\section{Introduction}

Stuttering is a serious communication disorder, characterized by involuntary disruptions in speech, and it significantly affects or impedes the capacity of individuals to communicate efficiently (1). A higher prevalence rate of stuttering $(\sim 1.4 \%)$ is reported in children and adolescents aged (2-19) years old (2).

The occurrence of stuttering may be related to cellular physiological, genetic, neurological and psychological disorders, and this is significantly affected by environmental factors (3-5). For example, in previous studies, in $71 \%$ of patients who stuttered, their condition could be attributed to genetic factors, whereas in the other $29 \%$ of patients, their condition could be attributed to environmental factors (6). Stuttering has been shown to significantly affect behavioral, cognitive, psychological and social aspects of a patient $(3,4)$. In the preadolescent population, $4-5 \%$ of individuals exhibit stuttered speech, whereas in the adult, this decreases to $\leq 1 \%$ of individuals (7-10).

Previous studies proposed that the neuropathophysiology of stuttering may involve deficiencies in connectivity amongst the cortical networks of regions that normally allows left-sided engagement of the inferior frontal and premotor cortices for efficient planning and execution of sound production, and have also suggested that this deficiency in connectivity of the left premotor/motor areas may be related to differences in the right hemisphere and subcortical activity in individuals who stutter (11). Stuttering neurophysiology has been shown to be linked with other non-speech related cognitive functions. For example, perception of speech is known to activate the same sensorimotor mechanisms that are involved in speech production (12-14), with activation levels that typically correlate with task difficulty (15). This leads to an increase in sensorimotor activity, which appears to be associated with cognitive resource allocation (e.g. attention and working memory) and subsequently more difficult tasks, such as discriminating specific speech in a noisy environment (16). In addition, a recent review provided a valuable means of linking the cognitive differences associated with stuttering to the underlying deficits that impact speech (17). 
Previous studies have shown that the core symptoms of stuttering required abnormal function of the neural circuits, whereas the individual manifestations of stuttering behavior can vary (18-21). However, other factors, such as emotional reactions and motor compensatory behaviors and therapy, may interact with core neural deficits. For example, stuttering severity can be exacerbated by greater demands on attention, linguistic complexity and emotional significance of the speech context (22).

Spontaneous normal recovery from stuttering ranges from $40-80 \%$ in children $<10$ years old (23), and in some studies, up to $89 \%$ of children who stutter recovered completely by adulthood (24). Adolescence is a unique period in life, marking the transition from childhood to adulthood, when individuals undergo physiological and physical maturation, and this period of life is associated with complex cognitive and social growth (25). It is conceivable that the additional burden of a stutter in this complicated period of development may increase the level of stress and negative psychological states of individuals (26). Treating stuttering amongst adolescents is likely to remove the additional associated-negative psychological effects.

Adolescents and older adults who stutter exhibit more severe negative psychological states, particularly depression and anxiety, and score lower on mental health assessments (24,27-29). Several studies have reported an association between cellular physiological changes in the levels of stress hormones, particularly in diseases associated psychological disorders (30-32).

Different treatment approaches for stuttering have shown to be most amenable for younger aged individuals compared with individuals in adulthood who stutter. This may be related to the greater degree of neuronal plasticity in individuals of a younger age compared with the less tractable and far less responsive to treatment neurons of adults, for whom it has often been a long-term problem (27,33-35).

Although positive clinical results with traditional drugs, such as biperiden, clomipramine and haloperidol have been reported $(36,37)$, the management of stuttering is difficult and most of the times frustrating. In addition, several therapeutic trials based on an improvement in speech fluency, means of communication or targeting cognitive, behavioral or psychologically associated factors provided limited information regarding the exact mechanisms underlying the cause of stuttering $(27-29,34,35)$.

Alternative medicine strategies based on the use of herbal medicines to treat stuttering or related consequences such as depression, anxiety and other mental health problems are poorly represented in the field of stuttering therapeutics.

Green tea (Camellia sinensis), is one of the most popular beverage drinks consumed worldwide (36-38). Epidemiological studies suggested that green tea consumption may be associated with several beneficial effects on human health (36-38). It has been shown to beneficial in the management of several diseases, such as adiposity, metabolic disorders, cardiovascular diseases and cancer $(36,38)$. Additionally, green tea has been to shown to exert beneficial effects on an individual's psychological state. It also possesses antioxidant, antimicrobial and anti-inflammatory activities due to the polyphenolic compounds present in green tea, such as theanine (also known as $\gamma$-glutamylethylamide) a novel amino acid present in green tea that is significantly associated with relaxation and a reduction in cognitive stress, depression and anxiety amongst humans (39-43).

Recently, the safety, tolerability and pharmacokinetic properties of the administration of EGCG as a single dose (ranging from 50-1,600 mg) were assessed. The results showed that administration of $>1 \mathrm{~g}$ of purified EGCG or in green tea has a mean terminal elimination half-life $(\mathrm{t} 1 / 2 \mathrm{z})$ of $1.9-4.6 \mathrm{~h}$. The data showed that doses of purified EGCG $\leq 1,600 \mathrm{mg}$ were generally well tolerated (44). Hepatotoxicity of high doses of purified EGCG intake or decaffeinated green tea were more pronounced in subjects with a high body mass index (BMI), or when green tea extracts where used in food supplements for weight control (45). In addition, it was reported that consumption of $\geq 5$ cups/day green tea did not elevate alanine transferase (ALT) levels, a measure of hepatotoxicity (45). Catechins from green tea, prepared in the traditional manner, as well is reconstituted drinks with an equivalent composition to traditional green tea infusions were, in general, considered safe. However, in rare cases, green tea infusions have been shown to induce minor harmful effects on the liver (45).

Epidemiological studies have identified an association between caffeine intake and mental health stress and lifestyle $(22,46,47)$. However, little is known regarding the effect of green tea supplements on stuttering and/or the related psychological consequences. Thus, in this study, the potential protective role of green tea, as a natural source of polyphenols, on stuttering or its related mental health consequences in adolescents with moderate stuttering (MS) were assessed. In addition, the physiological profile of stress hormones as a measure of the response to green tea was measured amongst participants.

\section{Materials and methods}

Participants. A total of 60 school students (median age, 12.1; age range, $12-18$ years old; female/male, 9/51) who following the same academic curricula were selected from different public schools and randomly invited to participate in this study. A sample size of 60 was selected from the public schools to give an estimated power of $96 \%$ and a significance level of 0.05 , with an expected frequency of $8.3 \%$. For all participants, to estimate the severity of the stuttering, one standardized questionnaire (depression anxiety stress scale-42; DASS-42) and one standardized test material (Stuttering Severity Instrument, 4th Edition; SSI-4) were used as previously reported (48-50) Then, the participants were clearly diagnosed by an Expert Speech Language Pathologist with $>15$ years of experience in his respective field, and the students were classified into two groups; normal healthy group $(n=30)$ and a MS group $(n=30)$. None of the selected participants had any chronic diseases, an abnormal iron status, iron or vitamin deficiency, or mental diseases that could prevent them from participating in this study. In addition, participants classed as obese (BMI $\geq 25)$ or who received prescribed drugs (including hormonal contraceptives), or who had medical or psychiatric disorders that may cause or exacerbate the stuttering, or a negative emotional state were excluded from this study. All subjects were free of neurological or medical disorders. None of the studied 
Table I. Demographics and baseline characteristics of the participants ${ }^{c}$.

\begin{tabular}{lcccc}
\hline Parameters & Control group & Moderate stuttering group & Degree of freedom & $\mathrm{t}(\mathrm{P}-\mathrm{value})$ \\
\hline $\mathrm{N}$ & 30 & 30 & - & - \\
$\mathrm{Male} /$ female & $25 / 5$ & $26 / 4$ & - & - \\
Age, years, & $12.3 \pm 4.1$ & $11.9 \pm 5.8$ & 198 & $-0.231(0.82)$ \\
Body mass index, $\mathrm{kg} / \mathrm{m}^{2}$ & $22.8 \pm 3.2$ & $23.7 \pm 1.8$ & 198 & $-1.57\left(0.01^{\mathrm{a}}\right)$ \\
Waist-hip ratio & $0.82 \pm 0.09$ & $1.38 \pm 0.05$ & 198 & $-26.1\left(0.001^{\mathrm{b}}\right)$ \\
\hline
\end{tabular}

${ }^{\mathrm{a}} \mathrm{P} \leq 0.01,{ }^{\mathrm{b}} \mathrm{P} \leq 0.001 .{ }^{\mathrm{c}} \mathrm{Data}$ are presented as the mean $\pm \mathrm{SD}$.

subjects were under active behavioral or pharmacological stuttering therapy at the time of study participation. During the entire study, the supervision and follow up of all participants was performed at an outpatient clinic for rehabilitation: The Medical Rehabilitation Department, King Khalid University. The aims and methodology of this study as well as a complete description of the potential effects of green tea were explained to all participants. Informed consent was obtained from the parents of all students recruited in the present study. Research involving human subjects complied with all relevant national regulations and institutional policies, and were approved by the Review Board (HAPO-06-B-001) of The Deanship of Scientific Research, King Khalid University (Abha, Saudi Arabia; approval no. ECM\#2019-117). All studies complied with the guidelines described in the 1975 Declaration of Helsinki $(51,52)$. The demographic and baseline data of the participants are shown in Table I.

Assessment of stuttering severity. The SSI-4 quantifies disfluency duration, frequency and physical features in preschool children through to adulthood (48-50). The SSI-4 enables the assessment of behavioral severity levels in both readers and non-readers. Classification of stuttering severity based on the total score and percentile ranks was as follows: No (total score, 10-17; percentile rank, 1-11), mild (total score, 18-24; percentile rank, 12-40), moderate (total score, 25-31; percentile rank, 41-77), severe (total score, 32-36; percentile rank, 78-95), and very severe (total score, 37-46; percentile rank, 96-99) (48-50).

Assessment of green tea phenolic compounds. Commercial decaffeinated green tea bags produced by Kao Ltd., were purchased from a convenience store. Green tea leaves (tea bags) were analyzed for the presence of catechins and caffeine constituents in green tea (100 $\mathrm{mg}$ ) according to previously reported preparation techniques $(53,54)$.

A HP1050 Knauer liquid chromatograph equipped with a 200x4.6 mm, dp $5 \mu \mathrm{m}$, Hypersil ODS column, a UV-visible detector $(230 \mathrm{~nm})$, and a Rheodyne injector with a $20-\mu 1$ loop was used to estimate the contents of catechins and caffeine constituents in the leaves of decaffeinated green tea bags. A total of $5 \mu \mathrm{l}$ aqueous extract was injected. Total run time for the separation was $15 \mathrm{~min}$ at a flow rate of $0.60 \mathrm{ml} / \mathrm{min}$ and the mobile phase yielded results of $40 \%$ methanol: $60 \%$ distilled water. The phenolic contents of the extracts were determined by use of calibration plots constructed for every standard. Finally, the peaks of each phenolic content was
Table II. Daily dosage of catechins from green tea ${ }^{\mathrm{a}}$.

\begin{tabular}{lcccc}
\hline $\begin{array}{l}\text { Phenolic compound, } \\
\text { mg }\end{array}$ & 1 cup & 4 cups & 5 cups & 6 cups \\
\hline EGCG & 96 & 396 & 476 & 548 \\
EGC & 54 & 218 & 287 & 332 \\
ECG & 39 & 156 & 210 & 238 \\
EC & 31 & 128 & 156 & 189 \\
Total catechin $^{\mathrm{b}}$ & 256 & 986 & 1350 & 1536 \\
Theanine $^{\text {The }}$ & 0.03 & 0.13 & 0.16 & 0.18 \\
\hline
\end{tabular}

${ }^{\mathrm{a}} 1$ cup $=300 \mathrm{ml}$. ${ }^{\mathrm{b}}$ Total catechin concentration was defined as the sum of EGCG, EGC, ECG, and EC values. EC, epicatechin; ECG, epicatechin gallate; EGC, epigallocatechin; EGCG, epigallocatechin gallate.

identified and measured at $520 \mathrm{~nm}$. For all the phenolic acids, calibration plots were linear in a range of $0.0625-1 \mathrm{mg} \mathrm{ml}-1$ $\left(\mathrm{R}^{2}>0.9980\right)(53,54)$.

Preparation and uses of green tea supplement. Previous research studies have showed that individuals who drank 2-3 cups of green tea a day benefitted the most from the full antioxidant benefits of the polyphenols in green tea (53). However, in order to drink more cups of tea (6-8), decaffeinated green tea was used to prevent any effects of caffeine present in green tea leaves $(54,55)$, and 5-7 cups of decaffeinated green tea a day significantly promoted cardiovascular and metabolic health (56). Thus, in the present study, decaffeinated green tea bags were steeped in boiled water (300 $\mathrm{ml} / \mathrm{cup}$ ) for $10 \mathrm{~min}$. No sugar or milk was added to the tea, but artificial sweetener was used in accordance with the preference of the participants. The contents of polyphenols per each cup of green tea are provided in Table II. In this study; participants were provided with 6 cups of green tea in a container and were asked to consume the entire amount within 6-8 h every day for 6 weeks. From previous studies that recommend that administration of $>5$ cups of decaffeinated green tea, which contained a total phenolic compound dose of 50-1,600, it was shown that these doses were well tolerated and did not have any notable side effects $(44,45,53-56)$. Thus, in the present study, it was proposed that administration of 6 cups of decaffeinated green tea bags was safe and may have 
Table III. Hepatotoxic and renal effects of administration of drinking six cups of green tea/day for 6 weeks ${ }^{\mathrm{a}}$.

\begin{tabular}{|c|c|c|c|c|c|c|}
\hline \multirow[b]{2}{*}{ Parameters } & \multicolumn{3}{|c|}{ Control group } & \multicolumn{3}{|c|}{ Moderate stuttering group } \\
\hline & Before & After & P-value & Before & After & P-value \\
\hline AST, U/l & $13.8 \pm 3.6$ & $13.9 \pm 2.9$ & 0.142 & $14.3 \pm 2.5$ & $14.5 \pm 1.9$ & 0.18 \\
\hline ALT, U/1 & $11.5 \pm 5.7$ & $12.6 \pm 5.2$ & 0.381 & $13.3 \pm 2.5$ & $13.7 \pm 2.8$ & 0.36 \\
\hline Total-bilirubin & $0.45 \pm 0.12$ & $0.50 \pm 0.34$ & 0.145 & $0.52 \pm 0.15$ & $0.58 \pm 0.18$ & 0.16 \\
\hline Creatinine & $0.57 \pm 0.18$ & $0.62 \pm 0.12$ & 0.134 & $0.61 \pm 0.21$ & $0.68 \pm 0.31$ & 0.14 \\
\hline
\end{tabular}

${ }^{\text {aData }}$ are presented as the mean $\pm \mathrm{SD}$. AST, aspartate transferase; ALT, alanine transferase. Normal values of AST are $\leq 40 \mathrm{U} / \mathrm{l}$ of ALT are $\leq 41 \mathrm{U} / \mathrm{l}$, of total-bilirubin are $\leq \mathrm{g} . \mathrm{dl}$ and of creatinine are $\leq 1.2 \mathrm{~g} / \mathrm{dl}$.

considerable potency for improving the negative emotional states of participants. In addition, the HPLC results showed that 6 cups of decaffeinated green tea had a safe amount of EGCG $(548 \mathrm{mg})$ and total catechin content $(1,536 \mathrm{mg})$ (Table III). To avoid any changes in the proper constituents of green tea, participants were told not to reheat the tea during the day, and to drink it straight from the container $(54,55)$. Although all participants had no iron disorders or anemia, they were advised to wait at least $1 \mathrm{~h}$ after eating before drinking green tea to avoid the influence of green tea on iron absorption and iron status. During the entire study period, all participants were under medical observation by qualified medical doctors to detect any hepato- or renal cytotoxicity as a result of green tea consumption. All participants were subjected for the routine analysis of serum aspartate transferase (AST), alanine transferase (ALT), total bilirubin and creatinine levels following treatment with tea (six cups/6 weeks). In addition, the participants were instructed not to change their normal habits or use any other soft or hot drinks containing caffeine or drugs that may have affected the results during the period of study.

Assessment of negative emotional states. All participants were subjected to the estimation of negative emotional states preand post-green tea administration (six cups/day for 6 weeks). Before and after green tea administration, all participants were under careful follow up by an Expert Speech Language Pathologist with $>15$ years of experience in their respective field. The initial changes in the scores of depression, anxiety, stress and mental health, as measures of the individuals psychological state, were evaluated before and after green tea supplementation as follows.

Assessment of depression, anxiety and stress. All participants underwent estimation of negative emotional states of depression, anxiety and stress using DASS, as previously reported (56). The DASS questionnaire consists of 42 items designed to assess three related undesirable emotional states: Depression, anxiety and tension/stress (56). Each item has a four-point rating scale: Did not apply to me at all (score 0)-to applied to me very much (score 3 ). DASS has 14 items each for depression, anxiety and stress $(14+14+14=42)$. The score for each of the respondents over each of the sub-scales (depression, anxiety and stress) were then estimated as per the severity-rating index: Normal, mild, moderate, severe and extremely severe (56).

Assessment of mental health status. Mental health status was evaluated in all participants using the 12-item General Health Questionnaire (GHQ 12) as previously reported (42,57). In general practice, the GHQ 12 is recommended as a screening tool for assessing mental ill-health $(42,56)$. In this questionnaire, responses to each item were coded on (0-0-1-1). The sum of all the questions is the final GHQ score, and is in an integer between 0-12. Participants with a GHQ score $\geq 4$ are considered to have mental ill-health problems $(58,59)$.

Assessment of adrenal hormones. Blood samples were taken once from for all participants at 8.30 AM pre and post green tea consumption (all 6 cups). After centrifugation, serum samples of all tests were stored at $-80^{\circ} \mathrm{C}$ until required. Several adrenal hormones were measured in order to detect several major adrenal pathways. Both cortisol and acetylcholine (ACTH) were measured in serum samples using RIA-ELISA (cat. no. KIPI28000; DPC Inc.) and SIA-ELISA kits (cat. no. M046006; MD Biosciences Inc.), respectively according to the manufacturer's protocol. Serum levels of DHEA were measured using an immunoassay according to the manufacturer's protocol (cat. no. DHA31-K01; IMMULITE 1000, Diagnostic Products Corporation), with an intra-assay coefficient of variation of $<5.2 \%$. Serum levels of corticosterone were measured using a quantitative competitive enzyme immunoassay technique that measures corticosterone levels according to the manufacturer's protocol (cat. no. EC3001-1; Assay pro LLC.).

Statistical analysis. Statistical analysis was performed using SPSS version 17 (SPSS Inc.). Data are presented as the mean \pm SD. For all continuous variables, such as the levels of stress hormones, and the renal and hepatotoxicity parameters, a paired t-test was used. A Wilcoxon signed rank sum test was performed for non-parametric data (depression, anxiety, stress and mental health scores). Pearson's correlation analysis was performed to calculate the correlations between the levels of adrenal hormones and the scores of stuttering, stress, anxiety and depression in adolescents with moderate stuttering. $\mathrm{P}<0.05$ was considered to indicate a statistically significant difference. 
Table IV. Improvement of stuttering and mental status in adolescents with moderate stuttering before and after the green tea regimen ${ }^{\mathrm{c}}$.

\begin{tabular}{lcccccccc}
\hline & \multicolumn{2}{c}{ Stuttering and psychological status } & & \multicolumn{3}{c}{ Clinical response } \\
\cline { 2 - 3 } Parameters & Before & After & P-value & & Before & After & P-value \\
\hline Depression score & $22 \pm 3$ & $11 \pm 2^{\mathrm{b}}$ & $<0.001^{\mathrm{b}}$ & & Moderate & Mild $^{\mathrm{b}}$ & $<0.001^{\mathrm{b}}$ \\
Stress score & $24 \pm 5$ & $10 \pm 2^{\mathrm{b}}$ & $<0.001^{\mathrm{b}}$ & & Moderate & Mild $^{\mathrm{b}}$ & $<0.001^{\mathrm{b}}$ \\
Anxiety score & $15 \pm 3$ & $8 \pm 2^{\mathrm{a}}$ & $<0.01^{\mathrm{a}}$ & & Moderate & Mild $^{\mathrm{a}}$ & $<0.01^{\mathrm{a}}$ \\
Mental health scores & $6.8 \pm 3.5$ & $4.3 \pm 2.8^{\mathrm{a}}$ & $<0.01^{\mathrm{a}}$ & & Moderate & Mild $^{\mathrm{a}}$ & $<0.01^{\mathrm{a}}$ \\
Total stuttering score (F+DSP+PC) & $32 \pm 6$ & $23 \pm 5^{\mathrm{b}}$ & $<0.001^{\mathrm{b}}$ & & Moderate & & Mild $^{\mathrm{b}}$ & $<0.001^{\mathrm{b}}$
\end{tabular}

${ }^{\mathrm{a}} \mathrm{P}<0.01$ (before vs. after) for stuttering and psychological status following treatment. ${ }^{\mathrm{b}} \mathrm{P}<0.001$ (before vs. after) for clinical response of stuttering. ${ }^{C}$ Data are presented as the mean \pm SD. A Wilcoxon signed rank sum test was used for analysis of stuttering and psychological status. F, frequency; DSP, duration of speech behavior; PC, physical concomitants.

\section{Results}

In this study, the beneficial effects of green tea supplementation on stuttering and its associated negative psychological states were evaluated in adolescents. A total of 60 adolescents aged 12-18 years old of both sexes were enrolled in the present study, and classified using SSI-4 for estimation of the severity of the stuttering. The participants were classified into two groups; normal healthy group $(n=30)$ and the MS group $(n=30)$, as shown in Table I. The data showed that adolescents in the experimental group were significantly more obese, as measured by $\mathrm{BMI},(\mathrm{P}=0.01)$ and had a larger waist-hip ratio $(\mathrm{P}=0.001)$ compared with the healthy controls (Table I).

Assessment of active constituents in decaffeinated green tea bags. Green tea polyphenolic compounds were evaluated using liquid chromatography analysis as shown in Table II. The results showed that the total catechin content in one cup of decaffeinated green tea bags was $256 \mathrm{mg}$. The data showed that drinking six cups of green tea contained a total catechin content of $1,580 \mathrm{mg}$ as well as containing thiamine and other related polyphenols (Table II).

Assessments of the cumulative side effects of consumption of six cups of decaffeinated green tea. The cumulative side effects of administration of six cups of decaffeinated green tea containing EGCG and other catechins for 6 weeks on both the liver and kidneys were estimated (Table III). The results showed no hepato- or renal toxicity in all participants following administration of decaffeinated green tea. In this experiment, the concentrations of serum AST, ALT, total bilirubin and creatinine did not increase notably following treatment with tea (six cups/6 weeks). The data obtained after 6 six did not differ significantly from that obtained prior to initiation of the study (Table III).

Assessment of the effects of green tea on depression, anxiety and stress. The correlation between negative psychological states and the incidence of stuttering amongst adolescents was evaluated using DASS and GHQ 12 tests (Fig. 1). The data showed that there was a significant reduction in the DASS scores for depression (Fig. 1A), anxiety (Fig. 1B) and stress (Fig. 1C), as well as a decrease in GHQ-12 scores (Fig. 1D) for mental health in both control $(\mathrm{P}=0.01)$ and adolescents with MS $(\mathrm{P}=0.001)$ at the end of the study procedure. The data showed that the administration of six cups of green tea for 6 weeks significantly improved depression, anxiety, stress and mental health status amongst adolescents with MS whilst also improving the mental health status of healthy subjects as well. As show in Table IV, a significant improvement in the scores of depression, anxiety, stress and mental health status were observed among adolescents with MS. Depression, anxiety, stress and mental health status among subjects with MS were significantly reduced.

Assessment of the effects of green tea on stuttering. The effect of green tea supplement on the severity of stuttering among adolescents was evaluated using the SSI-4 test (Fig. 2). Frequency, duration of speech behavior and physical concomitants as measures of stuttering showed significant improvements in the control subjects $((\mathrm{P}=0.01$; Fig. $2 \mathrm{~A})$ as well as in the adolescents with MS ( $\mathrm{P}=0.001$; Fig. $2 \mathrm{~B})$ following the 6 weeks of green tea consumption. In addition, a positive clinical response to green tea supplementation was reported in the adolescents with MS. The data showed a significant reduction in the total score of the SSI-4 test to a mild status (Table IV), indicating the probable positive curative effects of green tea against stuttering.

Assessment of the effects of green tea on adrenal stress hormones. To study the relationship between the physiological response of adrenal stress hormones with both stuttering and a negative psychological status, the expression levels of cortisol, DHEA, ACTH, corticosterone and the cortisol:DHEA ratio were assayed in all participants (Table V; Fig. 3). All subjects in the control group (Fig. 3A) and MS group (Fig. 3B) showed a significant decrease $(\mathrm{P}=0.01$ and $\mathrm{P}=0.001$, respectively) in the expression levels of cortisol, DHEA, ACTH and corticosterone, with an increase in the cortisol:DHEA ratio following the 6 weeks of green tea administration.

Together, the results highlight the beneficial effects of decaffeinated green tea on stuttering and mental health. In addition, the changes in expression of adrenal stress hormones were significantly associated with the improvements in 
depression, anxiety, stress and mental health status amongst adolescents who stuttered (Table V).

\section{Discussion}

In this study the administration of six cups/day of green tea for 6 weeks significantly reduced depression, anxiety, stress and the mental health consequences associated with stuttering in adolescents. The severity of stuttering was significantly reduced to a mild status amongst adolescents who suffered from MS.

Approximately 4-5\% preadolescents suffer from some degree of stuttering compared with $\leq 1 \%$ in adults $(9,33,35)$, and this has compounding effects on the behavioral, cognitive, psychological and social aspects for a patient (2-4). In addition, more severe negative psychological states are significantly more commonly reported in adolescents and adults who stutter, particularly in relation to mental health $(31,32,39)$.

In this study, depression, anxiety, stress and mental health status were significantly reduced in both the control group and adolescents who stuttered following green tea supplementation. The data significantly correlated with the reduction in the severity of stuttering from moderate to mild, highlighting the significant improvement in clinical response to green tea. This indicates an interesting association between green tea consumption in everyday life and mental health problems associated with stuttering. It has been widely reported that mental stress, anxiety and depression have a significant influence on the psychological status of people who stutter $(3,4,47-54)$. Previous studies showed that the polyphenolic compounds present in green tea, particularly catechins and theanine, enhanced relaxation in situations where an individual were in a negative mood, depressed or stressed (40-42,60,61).

Several studies have reported on the association between changes associated with cellular physiological changes and the levels of stress hormones, particularly in individuals with psychological disorders (38-41). Thus, in the present study, adrenal stress hormones, including cortisol, DHEA, ACTH, corticosterone and the cortisol:DHEA ratio were evaluated in the control and experimental group.

The levels of stress hormones were significantly improved and this was positively correlated with the reduction in the scores of stuttering and the related depression, anxiety, stress and mental health consequences. The data confirmed the potential protective role of green tea against the severity of stuttering, particularly among younger individuals. This may be due to the relaxing effect as well as other biological activities of the polyphenolic compounds present in green tea $(35,36,52-57)$. In experimental animals, green tea polyphenols have been shown to have a significant antidepressant-like effect on experimental animals $(40,41,60,61)]$. Green tea polyphenols have been shown to reduce serum corticosterone and ACTH levels in mice exposed to severe stressing factors. These studies showed that green tea polyphenols exert antidepressant-like effects in mouse behavioral models of depression, where the mechanism likely involves inhibition of the hypothalamic-pituitary-adrenal axis (58-61).

Recent clinical trials have shown that higher consumption of green tea significantly lowers the prevalence depression-like symptoms in elderly Japanese individuals $(60,61)$. In addition,

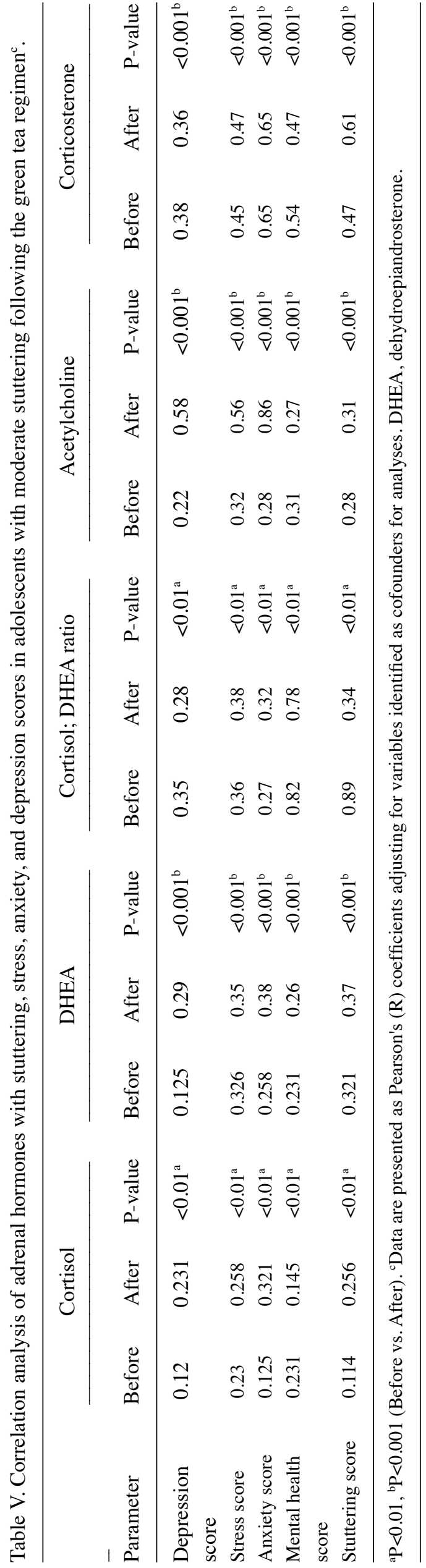



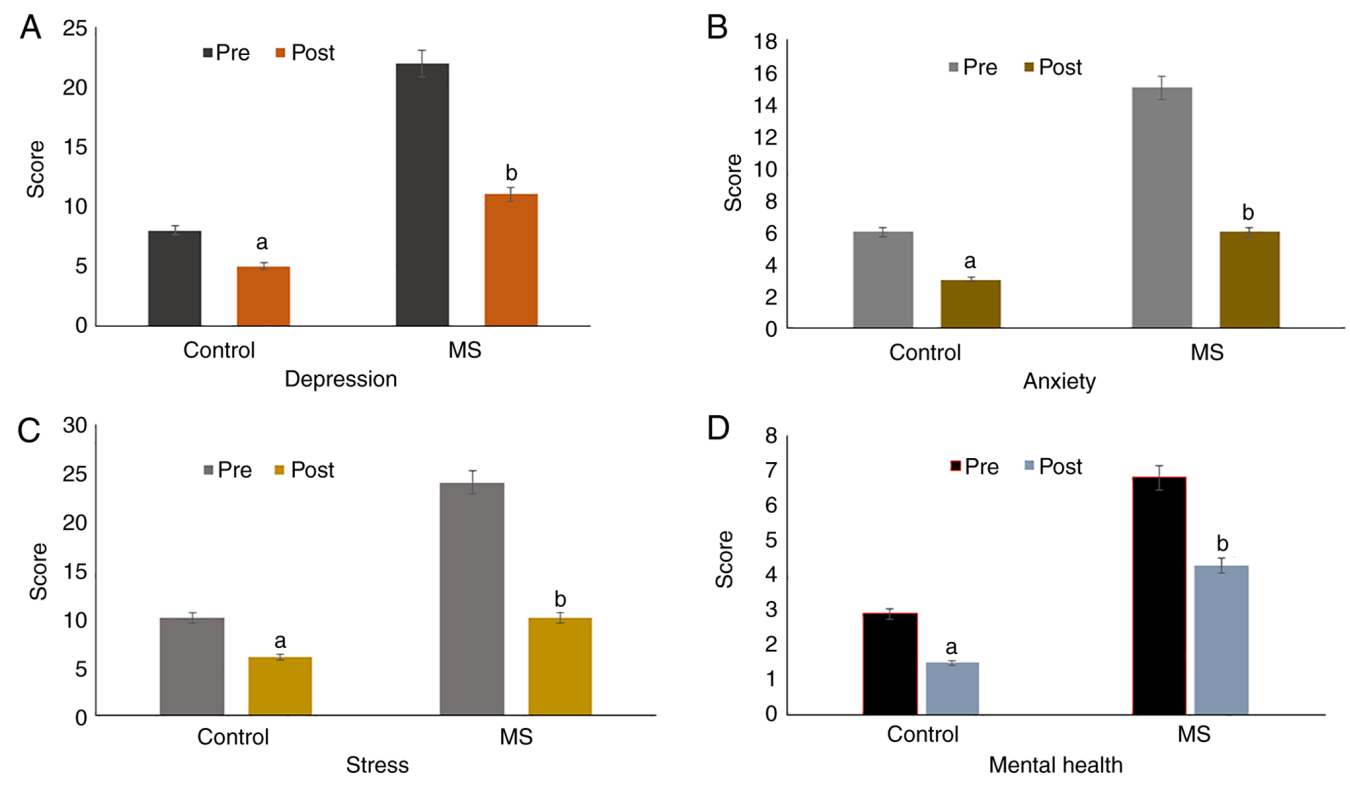

Figure 1. Graphical representation of the scores obtained for DASS and GHQ-12 scores for estimating the negative psychological states (A) depression, (B) anxiety, (C) stress and (D) and mental health in the control and MS patients pre- and post-green tea consumption (six cups/day for 6 weeks). The results showed a significant decrease in the negative psychological traits of depression, anxiety, stress and mental health status in the Control ( $\mathrm{P}=0.01$; pre vs. post) and adolescents with MS ( $\mathrm{P}=0.001$; pre vs. post). The results are presented as the mean $\pm \mathrm{SD}$. A Wilcoxon signed rank sum test was performed for analysis of non-parametric data, including depression, anxiety, stress and mental health scores. $\mathrm{P}<0.05$ was considered to indicate a statistically significant difference. DASS, depression anxiety stress scale; GHQ, General Health Questionnaire; MS, moderate stuttering.
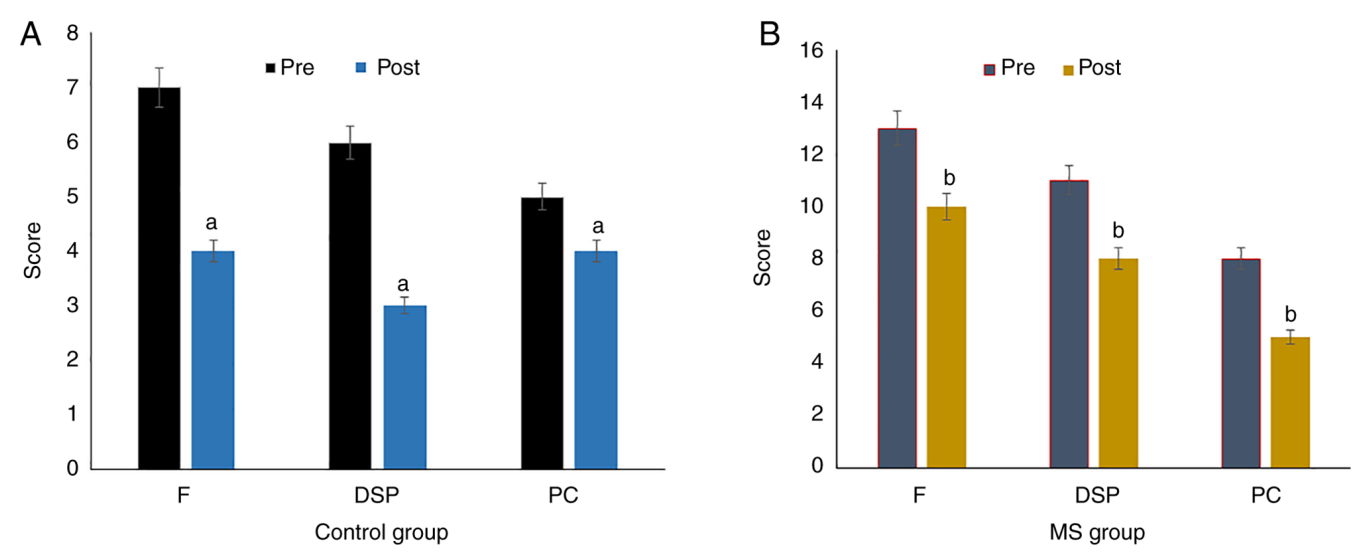

Figure 2. Graphical representation of the scores obtained for SSI-4 for estimating frequency, duration of speech behavior and physical concomitants as measures of stuttering in (A) the Control group and (B) adolescents with MS pre- and post-green tea supplement (six cups/day for 6 weeks). There was a significant decrease in stuttering scores amongst healthy Control subjects and adolescents with MS. The SSI-4 scores for estimating parameters of stuttering, such as DSP and PC were statistically analyzed using a Wilcoxon signed rank sum test. SSI-4, Stuttering Severity Instrument; MS, moderate stuttering; F, frequency; DSP, duration of speech behavior; PC, physical concomitants.
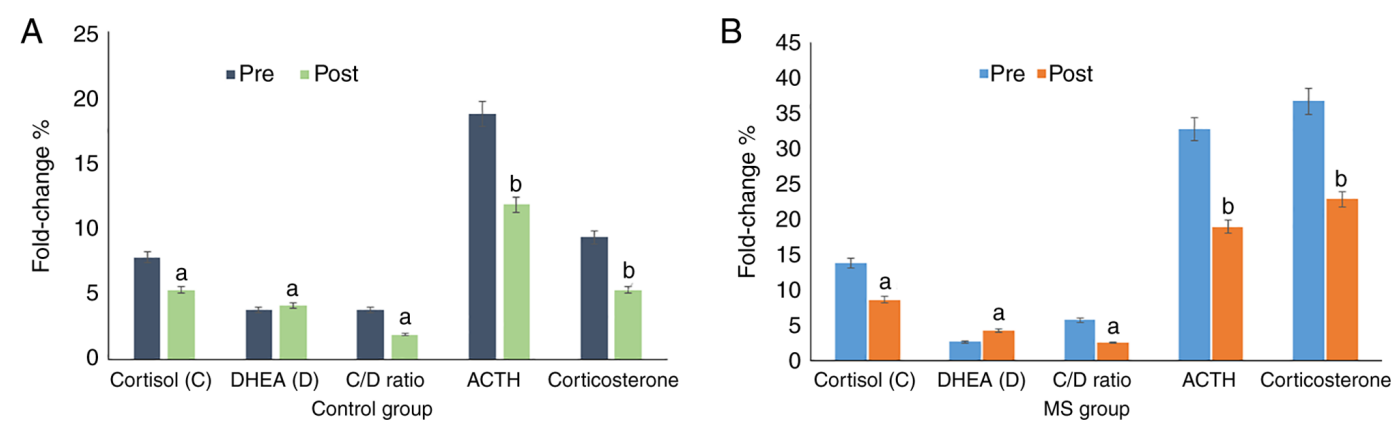

Figure 3. Levels of adrenal stress hormones in (A) the Control group and (B) adolescents with MS. The results showed a significant decrease in the expression of cortisol, DHEA, ACTH and corticosterone, and increase in the cortisol:DHEA ratio in the Control and MS groups following administration of green tea (six cups/day for 6 weeks). All continuous variables, including the parameters of adrenal stress hormones were analyzed using a paired t-test. MS, moderate stuttering; DHEA, dehydroepiandrosterone; ACTH, acetylcholine. 
it was reported that green tea polyphenols inhibit monoamine oxidase enzyme activity, consequently increasing monoamine levels in glial cells $(60,61)$. Conventional and newer antidepressants exert their effects predominantly through an increase in the synaptic concentrations of monoamines, indicating that monoamine systems play an essential role in the mechanism of action of antidepressants and the pathophysiology of depression (59,61-66).

In conclusion, the present study showed that green tea as a source of natural polyphenols improved psychological disorders associated with stuttering in adolescents. Higher consumption of green tea (six cups/day) significantly improved the severity of stuttering and the related negative psychological states; depression, anxiety, stress and general mental health status in adolescents with MS. In addition, the improvement in stuttering and other consequences correlated positively with the reduction in the expression levels of adrenal stress hormones. However, additional studies with larger cohorts are required for a more thorough evaluation of the biological effects of green tea on stuttering.

\section{Acknowledgements}

The authors are grateful to the Deanship of Scientific Research at King Khalid University, Abha, Saudi Arabia for funding this work through a Research Group Project under grant number (RGP2/205/43).

\section{Funding}

This project was funded by the Deanship of Scientific Research at King Khalid University (Abha, Saudi Arabia; grant no. RGP2/205/43). The funding body played no role in the study design, manuscript writing, or decision to submit the manuscript for publication.

\section{Availability of data and materials}

The datasets used and/or analyzed during the present study are available from the corresponding author on reasonable request.

\section{Authors' contributions}

AA and SAG conceived and designed the study, performed the practical work, analyzed the data, and wrote and edited the manuscript. AA and SAG have read and approved the final manuscript. AA and SAG confirm the authenticity of all the raw data.

\section{Ethics approval and consent to participate}

The aims and methodology of this study as well as a complete description of the potential effects of green tea were explained to all participants. Informed consent was obtained from the parents of all students recruited in the present study. Research involving human subjects complied with all relevant national regulations and institutional policies, and were approved by the Review Board (HAPO-06-B-001) of The Deanship of Scientific Research, King Khalid University (Abha, Saudi Arabia; approval no. ECM\#2019-117). All studies complied with the guidelines described in the 1975 Declaration of Helsinki.

\section{Patient consent for publication}

Not applicable.

\section{Competing interests}

The authors declare that they have no competing interests.

\section{References}

1. Bloodstein O and Ratner NB: A Handbook on Stuttering. 6th edition. Thomson Delmar, Clifton Park, NY, 2008.

2. Craig A, Hancock K and Cobbin D: Managing adolescents who relapse following treatment for stuttering. Asia Pac J Speech Lang Hear 7: 79-91, 2002.

3. Yaruss JS and Quesal RW: Stuttering and the international classification of functioning, disability, and health: An update. J Commun Disord 37: 35-52, 2004.

4. Guitar B: Stuttering: An Intergrated Approach to Its Nature and Management. Lippincott Williams \& Wilkins, Baltimore, MD, 2006.

5. Howell P: Signs of developmental stuttering up to age eight and at 12 plus. Clin Psychol Rev 27: 287-306, 2007.

6. Peters TJ and Guitar B: Stuttering: An Integrated Approach to Its Nature and Treatment. Williams and Wilkins, Maryland, MD, 1991.

7. Yairi E, Ambrose $\mathrm{N}$ and Cox $\mathrm{N}$ : Genetics of stuttering: A critical review. J Speech Hear Res 39: 771-784, 1996.

8. Månsson H: Childhood stuttering: Incidence and development. J Fluency Disord 25: 47-57, 2000.

9. Guitar B: Stuttering: An integrated approach to its nature and treatmen. Lippincott Williams \& Wilkins, 2013.

10. Weston CG and Riolo SA: Childhood and adolescent precursors to adult personality disorders. Psychiatr Ann 37: 114-120, 2007.

11. Chang SE, Horwitz B, Ostuni J, Reynolds R and Ludlow CL: Evidence of left inferior frontal-premotor structural and functional connectivity deficits in adults who stutter. Cereb Cortex 21: 2507-2518, 2011

12. Callan D, Callan A, Gamez M, Sato MA and Kawato M: Premotor cortex mediates perceptual performance. NeuroImage 51: 844-858, 2010.

13. Bowers A, Saltuklaroglu T, Jenson D, Harkrider A and Thornton D: Power and phase coherence in sensorimotor mu and temporal lobe alpha components during covert and overt syllable production. Exp Brain Res 237: 705-721, 2019.

14. Bowers A, Saltuklaroglu T, Harkrider A and Cuellar M: Suppression of the $\mu$ rhythm during speech and non-speech discrimination revealed by independent component analysis: Implications for sensorimotor integration in speech processing. PLoS One 8: e72024, 2013.

15. Szenkovits G, Peelle JE, Norris D and Davis MH: Individual differences in premotor and motor recruitment during speech perception. Neuropsychologia 50: 1380-1392, 2012.

16. Jenson D, Thornton D, Harkrider AW and Saltuklaroglu T: Influences of cognitive load on sensorimotor contributions to working memory: An EEG investigation of mu rhythm activity during speech discrimination. Neurobiol Learn Mem 166: 107098, 2019.

17. Jenson D, Bowers AL, Hudock D and Saltuklaroglu T: The application of EEG Mu rhythm measures to neurophysiological research in stuttering. Front Hum Neurosci 13: 458, 2020.

18. Chang SE, Garnett EO, Etchell A and Chow HM: Functional and neuroanatomical bases of developmental stuttering: Current insights. Neuroscientist 25: 566-582, 2019.

19. Chang SE: Research updates in neuroimaging studies of children who stutter. Semin Speech Lang 35: 67-79, 2014.

20. Lu C, Chen C, Ning N, Ding G, Guo T, Peng D, Yang Y, Li K and Lin C: The neural substrates for atypical planning and execution of word production in stuttering. Exp Neurol 221: 146-156, 2010.

21. Alm PA: Stuttering and the basal ganglia circuits: A critical review of possible relations. J Commun Disord 37: 325-369, 2004.

22. Smith A: Effects of caffeine on human behavior. Food Chem Toxicol 40: 1243-1255, 2002.

23. Ham RE: Therapy of Stuttering: Preschool Through Adolescence. Prentice-Hall, Englewood Cliffs, NJ, 1990.

24. Yairi E, Ambrose NG, Paden EP and Throneburg RN: Predictive factors of persistence and recovery: Pathways of childhood stuttering. J Commun Disord 29: 51-77, 1996. 
25. Spear LP: The adolescent brain and age-related behavioral manifestations. Neurosci Biobehav Rev 24: 417-463, 2000.

26. Kelly KB: Promoting adolescent health. Acta Paediatr 96: 1389-1391, 2007.

27. Lincoln M, Packman A and Onslow M: Altered auditory feedback and the treatment of stuttering: A review. J Fluency Disord 31: 71-89, 2006.

28. Lowe R, Guastella AJ, Chen NT, Menzies RG, Packman A, O'Brian S and Onslow M: Avoidance of eye gaze by adults who stutter. J Fluency Disord 37: 263-274, 2012.

29. Bricker-Katz G, Lincoln M and McCabe P: A life-time of stuttering: How emotional reactions to stuttering impact activities and participation in older people. Disabil Rehabil 31: 1742-1752, 2009.

30. Skosnik PD, Chatterton RT Jr, Swisher T and Park S: Modulation of attentional inhibition by norepinephrine and cortisol after psychological stress. Int J Psychophysiol 36: 59-68, 2000.

31. Nejtek VA: High and low emotion events influence emotional stress perceptions and are associated with salivary cortisol response changes in a consecutive stress paradigm. Psychoneuroendocrinology 27: 337-352, 2002.

32. Agha-Hosseini F, Mirzaii-Dizgah I, Mansourian A and Zabihi-Akhtechi G: Serum and stimulated whole saliva parathyroid hormone in menopausal women with oral dry feeling. Oral Surg Oral Med Oral Pathol Oral Radiol Endod 107: 806-810, 2009.

33. Prins $D$ and Ingham RJ: Evidence-based treatment and stuttering-historical perspective. J Speech Lang Hear Res 52: 254-263, 2009.

34. Prado-Velasco M and Fernández-Peruchena C: An advanced concept of altered auditory feedback as a prosthesis-therapy for stuttering founded on a non-speech etiologic paradigm. In: Handbook of research on personal autonomy technologies and disability informatics. IGI Global: pp76-118, 2011.

35. Blomgren M: Behavioral treatments for children and adults who stutter: A review. Psychol Res Behav Manag 6: 9-19, 2013.

36. Kumar A and Balan S: Fluoxetine for persistent developmental stuttering. Clin Neuropharmacol 30: 58-59, 2007.

37. Shaygannejad V, Khatoonabadi SA, Shafiei B, Ghasemi M, Fatehi F, Meamar R and Dehghani L: Olanzapine versus haloperidol: Which can control stuttering better? Int J Prev Med 4 (Suppl 2): S270-S273, 2013

38. Kobayashi K, Nagato Y, Aoi N, Juneja LR, Kim M, Yamamoto T and Sugimoto S: Effects of L-theanine on the release of $\alpha$-brain waves in human volunteers. Nippon Nogeikagaku Kaishi 72: 153-157, 1998 (In Japanese).

39. Maron DJ, Lu GP, Cai NS, Wu ZG, Li YH, Chen H, Zhu JQ, Jin XJ, Wouters BC and Zhao J: Cholesterol-lowering effect of a theaflavin-enriched green tea extract: A randomized controlled trial. Arch Intern Med 163: 1448-1453, 2003.

40. Jian L, Xie LP, Lee AH and Binns CW: Protective effect of green tea against prostate cancer: A case-control study in southeast China. Int J Cancer 108: 130-155, 2004.

41. Juneja LR, Chu DC, Okubo T, Nagato Y and Yokogoshi H: L-theanine-a unique amino acid of green tea and its relaxation effect in humans. Trends Food Sci Technol 10: 199-204, 1999.

42. Caspi A, Sugden K, Moffitt TE, Taylor A, Craig IW, Harrington H, McClay J, Mill J, Martin J, Braithwaite A and Poulton R: Influence of life stress on depression: Moderation by a polymorphism in the 5-HTT gene. Science 301: 386-389, 2003

43. Yagyu T, Wackermann J, Kinoshita T, Hirota T, Kochi K, Kondakor I, Koenig T and Lehmann D: Chewing-gum flavor affects measures of global complexity of multichannel EEG. Neuropsychobiology 35: 46-50, 1997.

44. Ullmann U, Haller J, Decourt JP, Girault N, Girault J, Richard-Caudron AS, Pineau B and Weber P: A single ascending dose study of epigallocatechin gallate in healthy volunteers. J Int Med Res 31: 88-101, 2003.

45. EFSA Panel on Food Additives and Nutrient Sources added to Food (ANS), Younes M, Aggett P, Aguilar F, Crebelli R, Dusemund B, Filipič M, Frutos MJ, Galtier P, Gott D, et al: Scientific opinion on the safety of green tea catechins. EFSA J 16 e05239, 2018.
46. Quinlan PT, Lane J, Moore KL, Aspen J, Rycroft JA and O'Brien DC: The acute physiological and mood effects of tea and coffee: The role of caffeine level. Pharmacol Biochem Behav 66: 19-28, 2000.

47. Loke WH: Effects of caffeine on mood and memory. Physiol Behav 44: 367-372, 1988

48. Kaplan GB, Greenblatt DJ, Ehrenberg BL, Goddard JE, Cotreau MM, Harmatz JS and Shader RI: Dose-dependent pharmacokinetics and psychomotor effects of caffeine in humans. J Clin Pharmacol 37: 693-703, 1997.

49. O'Brian S, Jones M, Packman A, Menzies R and Onslow M: Stuttering severity and educational attainment. J Fluency Disord 36: 86-92, 2011.

50. Riley GD and Bakker K: SSI-4: Stuttering severity instrument. Pro-Ed, 2009.

51. Declaration of Helsinki History Website. Ethical Principles For Medical Research. The JAMA Network. Retrieved 26 July 2015.

52. Vanderpool HY: The Ethics of Research Involving Human Subjects: Facing the 21st century. University Publishing Group, Inc., Frederick, ML, pp85, 1996.

53. Waksmundzka M, Oniszczuk A, Szewczyk K and Wianowska D Effect of sample-preparation methods on the HPLC quantitation of some phenolic acids in plant materials. Acta Chromatogr 19: 227-237, 2007.

54. Gabr SA, Alghadir AH, Ghoniem GA, Zhang XW, Choe YH, Park YJ and Kim BS: Regulation of cartilage and inflammatory biomarkers in rheumatoid arthritis patients treated with green tea therapy. Afr J Pharmacy Pharmacol 8: 263-273, 2014.

55. Pang J, Zhang Z, Zheng TZ, Bassig BA, Mao C, Liu X, Zhu Y, Shi K, Ge J, Yang YJ, et al: Green tea consumption and risk of cardiovascular and ischemic related diseases: A meta-analysis. Int J Cardiol 202: 967-974, 2016.

56. Kuriyama S, Shimazu T, Ohmori K, Kikuchi N, Nakaya N, Nishino Y, Tsubono Y and Tsuji I: Green tea consumption and mortality due to cardiovascular disease, cancer, and all causes in Japan: The Ohsaki study. JAMA 296: 1255-1265, 2006.

57. Wolfram S: Effects of green tea and EGCG on cardiovascular and metabolic health. J Am Coll Nutr 26: 373S-388S, 2007.

58. Lovibond PF and Lovibond SH: The structure of negative emotional states: Comparison of the depression anxiety stress scales (DASS) with the beck depression and anxiety inventories. Behav Res Ther 33: 335-343, 1995.

59. McDowell I and Newell C: Measuring health: A guide to rating scales and questionnaires. 2nd edition. University Press, New York, Oxford, pp225-237, 1996

60. Goldberg DP: The detection of psychiatric illness by questionnaire. London: Oxford University Press, 1972.

61. Niu K, Hozawa A, Kuriyama S, Ebihara S, Guo H, Nakaya N, Ohmori-Matsuda K, Takahashi H, Masamune Y, Asada M, et al: Green tea consumption is associated with depressive symptoms in the elderly. Am J Clin Nutr 90: 1615-1622, 2009.

62. Zhu WL, Shi HS, Wei YM, Wang SJ, Sun CY, Ding ZB and Lu L: Green tea polyphenols produce antidepressant-like effects in adult mice. Pharmacol Res 65: 74-80, 2012.

63. Mazzio EA, Harris N and Soliman KF: Food constituents attenuate monoamine oxidase activity and peroxide levels in $\mathrm{C} 6$ astrocyte cells. Planta Med 64: 603-606, 1998.

64. Haenisch B and Bönisch H: Depression and antidepressants: Insights from knockout of dopamine, serotonin or noradrenaline re-uptake transporters. Pharmacol Ther 129: 352-368, 2011.

65. Garrone G and Dick P: Monoamine oxidase inhibitors in the treatment of depressive states. Psychiatr Neurol (Basel) 140: 107-114, 1960 (In French).

66. Sagen J, Sortwell CE and Pappas GD: Monoaminergic neural transplants prevent learned helplessness in a rat depression model. Biol Psychiatry 28: 1037-1048, 1990.

This work is licensed under a Creative Commons Attribution-NonCommercial-NoDerivatives 4.0 International (CC BY-NC-ND 4.0) License. 